Research Article

\title{
Follow-Up in Bone Sarcoma Care: A Cross-Sectional European Study
}

\author{
Louren M. Goedhart $\mathbb{D}^{1},{ }^{1}$ Andreas Leithner $\mathbb{D}^{2}{ }^{2}$ Joris J. W. Ploegmakers $\mathbb{D}^{1}{ }^{1}$ \\ and Paul C. Jutte $\mathbb{B D}^{1}$ \\ ${ }^{1}$ Department of Orthopaedic Surgery, University of Groningen, University Medical Center Groningen, Groningen, Netherlands \\ ${ }^{2}$ Department of Orthopaedics and Trauma, Medical University of Graz, Graz, Austria
}

Correspondence should be addressed to Louren M. Goedhart; 1.m.goedhart@umcg.nl

Received 28 February 2020; Revised 17 May 2020; Accepted 15 June 2020; Published 1 July 2020

Academic Editor: Cornelis Verhoef

Copyright ( 92020 Louren M. Goedhart et al. This is an open access article distributed under the Creative Commons Attribution License, which permits unrestricted use, distribution, and reproduction in any medium, provided the original work is properly cited.

\begin{abstract}
Background. Follow-up of high-grade bone sarcoma patients with repeated radiological imaging aims at early detection of recurrent disease or distant metastasis. Repeated radiological imaging does expose (mostly young) patients to ionising radiation. At this point, it is not known whether frequent follow-up increases overall survival. Additionally, frequent follow-up subjects patients and families to psychological stress. This study aims to assess follow-up procedures in terms of frequency and type of imaging modalities in bone tumour centres across Europe for comparison and improvement of knowledge as a first step towards a more uniform approach towards bone sarcoma follow-up. Methods. Data were obtained through analysis of several follow-up protocols and a digital questionnaire returned by EMSOS members of bone tumour centres all across Europe. Results. All participating bone tumour centres attained a minimum follow-up period of ten years. National guidelines revealed variations in follow-up intervals and use of repeated imaging with ionising radiation. A local and a chest X-ray were obtained at $47.6 \%$ of the responding clinics at every follow-up patient visit. Conclusions. Variations were seen among European bone sarcoma centres with regards to follow-up intervals and use of repeated imaging. The majority of these expert centres follow existing international guidelines and find them sufficient as basis for a follow-up surveillance programme despite lack of evidence. Future research should aim towards evidence-based follow-up with focus on the effects of follow-up strategies on health outcomes, cost-effectiveness, and individualised follow-up algorithms.
\end{abstract}

\section{Introduction}

High-grade bone sarcomas are known as rare and aggressive malignancies with chondrosarcoma, osteosarcoma, and Ewing sarcoma as the most common entities [1]. Multimodal treatment including surgery and neoadjuvant therapy by an experienced multidisciplinary team is essential for survival $[2,3]$. Disease recurrences, local or metastatic, result in significant reduction of survival [4-9].

Follow-up through outpatient visits with radiological imaging is important to assess postoperative function and to detect local recurrent disease at an early stage. Follow-up is also useful to monitor surgical reconstruction as well as long-term cytotoxic effects of systemic therapy.
Osteosarcoma and Ewing sarcoma patients are relatively young though, which results in increased sensitivity to late stochastic effects of ionising radiation due to repeated radiological imaging [10-12]. Repeated follow-up visits raise healthcare expenses and may not lead to improved survival [13].

The most commonly used international guideline for follow-up is the ESMO-PaedCan-EURACAN Clinical Practice Guideline [14]. Based on a recent Asian singlecentre randomised study, a less intensive surveillance protocol in terms of frequency and imaging seems noninferior to a more intensive surveillance protocol in terms of survival after treatment of a sarcoma of the limb [15]. An American/ Canadian study group has described a large retrospective 
cohort with data on follow-up frequencies and timing of recurrences, and proposed an alternative (less intensive) follow-up schedule [13]. Based on these findings, more insight into follow-up procedures used in daily practice in Europe is valuable for comparisons. Regional or cultural differences may very well influence decision-making on follow-up procedures.

The European Musculoskeletal Oncology Society (EMSOS) aims to promote advance in science, disseminate knowledge, and promote mutual collaboration for bone sarcoma care between the different affiliated bone tumour centres. This observational cross-sectional study aims to assess follow-up procedures in terms of frequency and imaging modalities in several bone tumour centres all across Europe to compare and improve knowledge as a first step towards a more uniform approach of bone sarcoma follow-up.

\section{Material and Methods}

Data for this observational cross-sectional study were obtained from healthcare professionals. The authors formulated nine questions about organisation of care and produced a digital questionnaire using Google Forms (displayed in Appendix). The questionnaire was not validated. Representatives of EMSOS-affiliated bone tumour centres were approached by the authors based on the EMSOS member archive. We aimed for a proportional distribution across Europe in order to obtain a wide overview. The approached representatives who did return the questionnaire after one digital invitation and one digital reminder were acknowledged and specified as the EMSOS study group. All responses came from orthopaedic surgeons. Observational research among healthcare professionals does not fall under the scope of the Dutch Act on Medical Scientific Research Involving Human Beings (WMO). Data processing was performed using Microsoft Excel 2013 (United States), and analyses were performed using IBM SPSS Statistics for Windows (Version 23.0, United States).

\section{Results}

A digital questionnaire was sent to 54 EMSOS member representatives; we received a response of 17 representatives (31.5\%) from 12 different countries across Europe. The geographical dispersion across Europe of responding bone tumour centres is displayed in Figure 1.

Using the digital questionnaire as basis, all participating bone tumour centres use a protocol for oncological followup after treatment of high-grade bone sarcomas. The bases of these protocols are displayed in Table 1. Authorization for the oncological follow-up protocol was government-based in $33.3 \%$ and expertise-based in $66.6 \%$ of centres. The guideline as basis for the oncological follow-up protocol used differed across respondents. An international guideline (such as ESMO-PaedCan-EURACAN) was used by $66.6 \%$ of centres and a local/national guideline by $33.3 \%$. In terms of duration of oncological follow-up, all participating bone tumour centres attained a minimum of ten years. In two centres $(12.5 \%)$, the duration of oncological follow-up

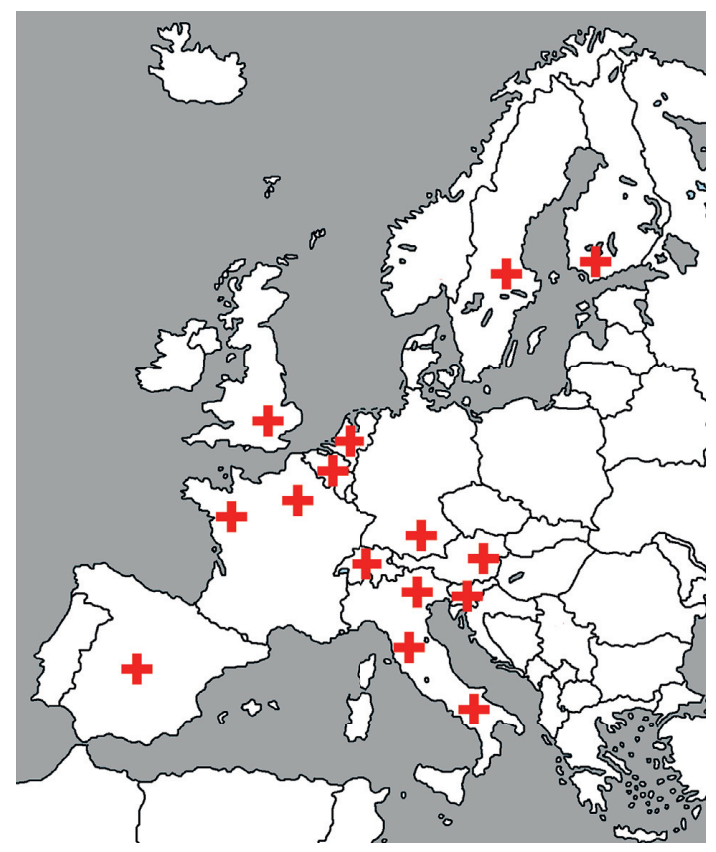

FIgURE 1: Geographical dispersion across Europe of responding bone tumour centres.

exceeded ten years. Separate sections and recommendations for osteosarcoma, chondrosarcoma, and Ewing sarcoma were seen in $62.5 \%$ of the respondents' follow-up protocol.

Regarding radiological imaging, a local X-ray only was performed during an oncological follow-up visit in one bone sarcoma centre (5.9\%). A local and chest X-ray was performed every follow-up visit in eight responding centres (47.6\%). In six of the responding centres (35.3\%), a local $\mathrm{X}$-ray was performed every follow-up visit with a chest X-ray at a different interval.

We received data points on follow-up intervals from 12 different countries for this study; variations in these intervals are displayed in Table 2. Finland has the shortest follow-up intervals with outpatient visits every two months for the first two years and then outpatient visits every four months up to five years postoperatively. The longest follow-up intervals are seen in the Netherlands, with an outpatient visit every four months between the first and second year of follow-up, downgraded to a follow-up interval of one year between two and five years postoperatively.

Lastly, respondents were asked for their opinion on several topics. Most respondents believe that early detection of a local recurrence as well as of a distant metastasis is important and of clinical relevance for additional treatment. However, some respondents emphasised that survival could depend more on the type and grade of the tumour than on early detection of recurrent disease. Only 25\% of respondents believe in added value of an additional, dedicated follow-up guideline for orthopaedic oncology, whereas the vast majority $(62.5 \%)$ believes that the current international ESMO guideline is sufficient. Differentiation between osteosarcoma, Ewing sarcoma, and chondrosarcoma in a follow-up guideline was found useful by $68.8 \%$ of respondents. 
TABLE 1: Baseline characteristics.

\begin{tabular}{|c|c|c|c|c|c|}
\hline & $\begin{array}{l}\text { No. of bone sarcoma } \\
\text { centres }\end{array}$ & $\begin{array}{l}\text { Authorization } \\
\text { basis }\end{array}$ & $\begin{array}{l}\text { Bone tumour } \\
\text { guideline }\end{array}$ & $\begin{array}{l}\text { Guideline as basis for oncological } \\
\text { follow-up protocol }\end{array}$ & $\begin{array}{l}\text { Minimum } \\
\text { follow-up }\end{array}$ \\
\hline The Netherlands & 4 & Government & National & International & 10 years \\
\hline Belgium & 5 & Expertise & Local & International & 10 years \\
\hline Germany & Not clear* & Expertise & National & International & 10 years \\
\hline $\begin{array}{l}\text { The United } \\
\text { Kingdom }\end{array}$ & 5 & Government & National & Local/national & 10 years \\
\hline France & 12 & Expertise & National & Local & 10 years \\
\hline Spain & 10 & Expertise & National & International & 10 years \\
\hline Italy & 10 & Expertise & Local & Local/national & 10 years \\
\hline Sweden & 3 & Government & National & International & 10 years \\
\hline Finland & 4 & Government & Local & International & 10 years \\
\hline Austria & 4 & Expertise & Local & International & 10 years \\
\hline Switzerland & 5 & Expertise & National & International & $>10$ years \\
\hline Slovenia & 1 & Expertise & National & National & $>10$ years \\
\hline
\end{tabular}

TABLE 2: Interval variations in the available follow-up protocols.

\begin{tabular}{|c|c|c|c|c|c|}
\hline & $\begin{array}{c}\text { Follow-up interval, } \\
0-1 \text { years }\end{array}$ & $\begin{array}{c}\text { Follow-up interval, } \\
1-2 \text { years }\end{array}$ & $\begin{array}{c}\text { Follow-up interval, } \\
2-4 \text { years }\end{array}$ & $\begin{array}{c}\text { Follow-up interval, } \\
4-5 \text { years }\end{array}$ & $\begin{array}{c}\text { Follow-up interval, } \\
5-10 \text { years }\end{array}$ \\
\hline ESMO guideline & 2-3 months & 2-3 months & 3-4 months & 6 months & 6 months \\
\hline The Netherlands & 3 months & 4 months & 12 months & 12 months & 12 months \\
\hline Belgium & 3 months & 3 months & 6 months & 6 months & 12 months \\
\hline Germany & 3 months & 3 months & 6 months & 12 months & 12 months \\
\hline The United Kingdom & 3 months & 3 months & 6 months & 6 months & 12 months \\
\hline France & 4 months & 4 months & 6 months & 6 months & 12 months \\
\hline Spain & 3 months & 3 months & 6 months & 6 months & 12 months \\
\hline Italy & 3 months & 3 months & 4 months & 6 months & 12 months \\
\hline Sweden & 3 months & 3 months & 6 months & 6 months & 12 months \\
\hline Finland & 2 months & 2 months & 4 months & 4 months & 12 months \\
\hline Austria & 3 months & 3 months & 3-6 months & 6 months & 12 months \\
\hline Switzerland & 3 months & 3 months & 3 months & 6 months & 12 months \\
\hline Slovenia & 3 months & 3 months & 6 months & 6 months & 6 months \\
\hline
\end{tabular}

\section{Discussion}

The aim of this observational cross-sectional study was to assess follow-up as a first step towards a more uniform approach of bone sarcoma follow-up. This study shows variation in follow-up protocols regarding frequency and use of imaging modalities. With the input from the EMSOS study group, we were able to gather valuable additional information and received several guidelines on oncological follow-up from across Europe.

Limitations for this publication are the observational nature of the study and the disproportional distributed participation from countries and centres. Furthermore, the questionnaire we used was not validated. Despite a digital invitation and reminder, we received a slightly disappointing response rate of $31.5 \%$. This might introduce response bias in this study. On the other hand, we did get a good general impression from most countries which may very well be representative for current policy, and the variation was clearly visible in our data. Regarding the displayed data, the follow-up intervals displayed are based on the input of the study group representatives and arranged by the country. In Germany, there is a known lack of consensus on authorisation of bone sarcoma centres. We, therefore, believe that Germany variability in follow-up intervals and imaging modalities between centres is likely.

As shown in the results, most of the participating bone tumour centres used an international guideline as basis for their national follow-up protocol, and the most commonly used being the ESMO-PaedCan-EURACAN Clinical Practice Guideline [14]. The National Institute for Health and Clinical Excellence (NICE) guideline is an extensive and evidence-based reference as well [16].

In general, follow-up surveillance programmes are based on the implication that early detection of recurrent disease or distant metastasis is of benefit to bone sarcoma patients.

Cool et al. evaluated the efficiency of their local follow-up surveillance programme for extremity bone sarcoma patients in a single-centre retrospective cohort study [17]. Regarding local recurrence, only $38 \%$ were detected with follow-up, whereas $62 \%$ of patients with a local recurrence presented with symptoms in between a follow-up interval. On the other hand, most pulmonary metastases (64\%) were detected using follow-up with repeated imaging while $36 \%$ of patients with pulmonary metastases were diagnosed outside the surveillance programme. 
The noninferiority trial of Puri et al. outlined that almost $90 \%$ of local recurrences are detected by patient themselves, stressing the importance of self-education [15]. Several small retrospective studies have described beneficial results from pulmonary metastasectomy in selected osteosarcoma patients. Beneficial prognostic factors were identified as small pulmonary metastases $(<2.0 \mathrm{~cm})$, less than five pulmonary metastases at diagnosis, and a relatively long disease-free interval (DFI) between primary disease and metastatic disease [18-20]. The DFI is an interesting parameter for closer analysis, and Yamamoto et al. associated a DFI $<12$ months with significantly lower overall survival compared to a DFI >12 months for patients eligible for primary pulmonary metastasectomy [19]. This means that for osteosarcoma patients, recurrence or metastasis within one year of surgical treatment is a negative prognostic factor. This is acknowledged by Cool et al., and their study showed that only $10 \%$ of patients with detected pulmonary metastasis survived [17]. In a subsequent study, Cool and Cribb followed 131 high-grade sarcoma patients. Metastatic disease developed in 15 patients, and only $13 \%$ was referred for metastectomy. This resulted in a prolonged disease-free survival, but curation was not achieved [21]. A retrospective cohort study from Kim et al. focused on postmetastatic survival. They found that the 5-year postmetastatic survival rate was $31 \%$ with a median length of 22 months. Local recurrence prior to metastasis, extrapulmonary metastasis, and poor histological response to preoperative chemotherapy were identified as negative prognostic factors [22]. In summary, the efficiency of follow-up surveillance programmes to detect local recurrences seems to be limited. Furthermore, the effects of intensive follow-up on overall survival remains controversial since early pulmonary metastasis results in inferior prognosis.

Regarding follow-up intervals, earlier detection of local recurrence facilitates the possibility for additional therapy which could lead to a longer subsequent survival period, but will the overall survival be affected? The follow-up interval advised by the ESMO for high-grade bone sarcomas is every 3 months for first two years after start of treatment. After two years, a follow-up interval of 4 months is advised from years 2 to 4 . Between 4 and 10 years, a follow-up interval of 6 to 12 months is recommended [14]. For this study, we received follow-up intervals from eleven countries that showed variation. The differences in follow-up intervals and use of repeated imaging as described in the results imply a lack of consensus, which reflects the lack of evidence. None of the responding bone sarcoma centres abide to the follow-up intervals after 2 years as advised in the ESMO guideline. This lack of consensus regarding follow-up intervals among experts for high-grade bone sarcomas is explicated in the 2018 ESMO guideline [14]. Furthermore, the authors of the NICE guideline state that, at the time of publication of their guideline, no comparative studies regarding follow-up strategies and the effects on health outcomes were found [16]. Gerrand et al. acknowledged that evidence is lacking for determination of optimal follow-up intervals [23]. However, Puri et al. found that a less intensive 6-month follow-up interval was noninferior to a 3-month interval in terms of recurrence-free survival and overall survival [15]. Furthermore, a recent retrospective cohort study by Cipriano et al. (including chondrosarcoma, osteosarcoma, and Ewing sarcoma) concluded that most cases of local recurrence occur within the first two years [13]. Late local recurrences (after four years) were uncommon. The highest rates of metastasis were also seen in the first two years for high-grade bone sarcomas with a ratio of 0.66 lung metastases per patient per year. After two years, metastases were seen at lower rates up to ten years. A ratio of 0.018 lung metastases per patient per year was seen 5-10 years after treatment. Based on their study, Cipriano et al. proposed a follow-up protocol for high-grade bone sarcomas. Follow-up should consist of a 3-month interval from 0-2 years, a 6-month interval between 3-4 years, and a 12-month interval from 5-10 years.

Regarding duration of follow-up, ten years was defined as final follow-up moment in $87.5 \%$ of the responding centres in this study. In an observational study by Marina et al., adult Ewing sarcoma survivors were compared with their siblings in terms of survival, cause-specific mortality, and chronic conditions [24]. This study with extended follow-up, up to 35 years after treatment, showed that the incidence of late mortality and subsequent neoplasms kept increasing over the years. Chronic cardiac and musculoskeletal conditions related to treatment (chemotherapy, radiation, and surgery) were also seen to increase after 10 years of follow-up. These findings support the need for a lifelong follow-up to assess the late effects of treatment.

In our study, variations were also seen in the use of imaging modalities as well as repeated imaging frequency based on the available guidelines. The ESMO guideline states that imaging of local recurrence or screening for distant metastases could be achieved with local imaging and chest $\mathrm{X}$-ray/CT scanning. Based on the data we obtained, some bone sarcoma patients had up to 10 low-dose chest CT scans in five years while others did not have a single scan. Puri et al. found that even though a CT scan facilitates an earlier diagnosis of pulmonary metastasis, the effects on recurrencefree survival and overall survival are not significantly different compared to a chest X-ray [15]. As mentioned earlier, repeated imaging during follow-up with ionising radiation has proven late stochastic effects in young bone sarcoma patients [10-12].

Several prognostic factors are known for chondrosarcoma, Ewing sarcoma, and high-grade central osteosarcoma. Metastasis at presentation, large primary tumour size, and tumours in the axial skeleton are associated with lower survival for Ewing sarcoma and high-grade central osteosarcoma [4, 5]. For chondrosarcoma, a high-grade tumour and axial localisation of the tumour are poor prognostic factors [6]. Based on these findings, we believe that such prognostic factors could be used to identify highrisk patients after primary treatment. Intensification of imaging during follow-up could be considered for these high-risk patients, despite the lack of evidence whether this will improve overall survival.

We believe that future research should elaborate on the effect of follow-up strategies on survival for comparison with 
the data presented by Puri et al. and Cipriano et al. $[13,15]$ Furthermore, cost-effectiveness of bone sarcoma follow-up is an interesting research perspective. Additionally, big data analysis could contribute to the development of an algorithm for individualised follow-up using known prognostic factors. For soft-tissue sarcomas, the PERSARC prediction model is an example to facilitate individualised follow-up [25].

In conclusion, variations were seen among European bone sarcoma centres with regards to follow-up intervals and use of repeated imaging. The majority of these expert centres follow existing international guidelines and find them sufficient as basis for a follow-up surveillance programme despite lack of evidence. Therefore, we believe that future research should aim towards evidence-based followup with focus on the effects of follow-up strategies on health outcomes, cost-effectiveness, and individualised follow-up algorithms.

\section{Abbreviations}

EMSOS: European Musculoskeletal Oncology Society

ESMO: European Society for Medical Oncology

NICE: The National Institute for Health and Clinical

Excellence

DFI: Disease-free interval.

\section{Appendix}

A nine-question digital questionnaire is used on organisation of bone sarcoma care:

(1) Do you use a protocol for oncological follow-up after treatment of high-grade bone sarcomas? (yes, no)

(2) If yes, what is this oncological follow-up protocol based on?

(i) Expert opinion/local guideline

(ii) National tumour guideline

(iii) International tumour guideline (e.g., ESMO guideline)

(3) According to your protocol, for how many years are bone sarcoma patients (OS, CS, and ES) monitored in terms of oncological follow-up? $(5,10$, and $>10$ years)

(4) Does your protocol contain separate sections/recommendations for OS, CS, or ES? (yes, no)

(5) Which radiological imaging tests are performed during an oncological follow-up visit?

(i) Only local X-ray

(ii) Local X-ray and chest X-ray

(iii) Local X-ray at every visit and chest X-ray at different interval

(6) In your opinion, how relevant is early detection of a local recurrence of high-grade bone sarcomas?

(7) In your opinion, how relevant is early detection of distant metastases (e.g., lung) from high-grade bone sarcomas?
(8) In your opinion, does a dedicated follow-up guideline for orthopaedic oncology have any added value?

(i) No, a local guideline is sufficient

(ii) No, a national guideline is sufficient

(iii) No, an international guideline (e.g., ESMO) is sufficient

(iv) Yes

(9) In your opinion, would a specific follow-up guideline for orthopaedic oncology have to differentiate between OS, CS, or ES? (yes, no).

\section{Data Availability}

The data used to support the findings of this study may be released upon request to the corresponding author via l.m.goedhart@umcg.nl.

\section{Disclosure}

The authors declare that they have no disclosures regarding this manuscript.

\section{Conflicts of Interest}

The authors declare that they have no conflicts of interest.

\section{Authors' Contributions}

Drs. Goedhart was involved in the study concept and design. Also, he was responsible for gathering, processing, and interpretation of the data well as preparing the manuscript. Prof. Leithner and Dr. Ploegmakers were involved in study design as well as reviewing the manuscript. Dr. Jutte was involved in the study concept and design as well as reviewing and editing the manuscript. The EMSOS study group was involved in generation of the data.

\section{Acknowledgments}

The authors would like to acknowledge and thank the EMSOS study group for participating and thereby facilitating this study. The authors also thank the representatives from the EMSOS study group: Michiel van de Sande, Leiden University Medical Center, Netherlands. Ingrid van der Geest, Radboud University Medical Center, Nijmegen, Netherlands; Hans Roland Dürr, the Medical Center of the University of Munich, Germany; Henrik Bauer, Karolinska Hospital, Stockholm, Sweden; François Gouin, University Hospital Hotel-Dieu, Nantes, France; Rob Pollock, Royal National Orthopaedic Hospital, Stanmore, UK; Blaz Mavcic, Ljubljana University Medical Centre, Slovenia; Pietro Ruggieri, University of Padova, Italy; David Biau, Hospital Cochin, Paris, France; Flavio Fazioli, Cancer Institute G. Pascal Foundation, Naples, Italy; Domenico Campanacci, Centro Traumatologico Ortopedico, Florence, Italy; Minna Laitinen, Helsinki University Central Hospital, Finland; Félix Shumelinsky, Jules Bordet Institute, Brussels, Belgium; 
Bruno Fuchs, Balgrist University Hospital, Zürich, Switzerland; and Eduardo Ortiz-Cruz, Hospital Universitario La Paz, Madrid, Spain.

\section{References}

[1] L. M. Goedhart, V. K. Y. Ho, P. D. S. Dijkstra et al., "Bone sarcoma incidence in the Netherlands," Cancer Epidemiology, vol. 60, pp. 31-38, 2019.

[2] H. Gelderblom, P. C. W. Hogendoorn, S. D. Dijkstra et al., "The clinical approach towards chondrosarcoma," The Oncologist, vol. 13, no. 3, pp. 320-329, 2008.

[3] B. Widhe and H. C. F. Bauer, "Surgical treatment is decisive for outcome in chondrosarcoma of the chest wall: a population-based Scandinavian sarcoma group study of 106 patients," The Journal of Thoracic and Cardiovascular Surgery, vol. 137, no. 3, pp. 610-614, 2009.

[4] K. R. Duchman, Y. Gao, and B. J. Miller, "Prognostic factors for survival in patients with Ewing's sarcoma using the surveillance, epidemiology, and end results (SEER) program database," Cancer Epidemiology, vol. 39, no. 2, pp. 189-195, 2015.

[5] K. R. Duchman, Y. Gao, and B. J. Miller, "Prognostic factors for survival in patients with high-grade osteosarcoma using the surveillance, epidemiology, and end results (SEER) program database," Cancer Epidemiology, vol. 39, no. 4, pp. 593-599, 2015.

[6] S. P. F. T. Nota, Y. Braun, J. H. Schwab, C. N. Van Dijk, and J. A. M. Bramer, "The identification of prognostic factors and survival statistics of conventional central chondrosarcoma," Sarcoma, vol. 2015, Article ID 623746, 11 pages, 2015.

[7] A. Angelini, G. Guerra, A. F. Mavrogenis, E. Pala, P. Picci, and P. Ruggieri, "Clinical outcome of central conventional chondrosarcoma," Journal of Surgical Oncology, vol. 106, no. 8, pp. 929-937, 2012.

[8] F. Fiorenza, A. Abudu, R. J. Grimer et al., "Risk factors for survival and local control in chondrosarcoma of bone," The Journal of Bone and Joint Surgery. British Volume, vol. 84-B, no. 1, pp. 93-99, 2002.

[9] S. E. Bosma, O. Ayu, M. Fiocco, H. Gelderblom, and P. D. S. Dijkstra, "Prognostic factors for survival in Ewing sarcoma: a systematic review," Surgical Oncology, vol. 27, pp. 603-610, 2018.

[10] M. S. Pearce, J. A. Salotti, M. P. Little et al., "Radiation exposure from CT scans in childhood and subsequent risk of leukaemia and brain tumours: a retrospective cohort study," The Lancet, vol. 380, no. 9840, pp. 499-505, 2012.

[11] N. M. Y. Journy, C. Lee, R. W. Harbron, K. McHugh, M. S. Pearce, and A. Berrington de González, "Projected cancer risks potentially related to past, current, and future practices in paediatric CT in the United Kingdom, 19902020," British Journal of Cancer, vol. 116, no. 1, pp. 109-116, 2017.

[12] J. D. Mathews, A. V. Forsythe, Z. Brady et al., "Cancer risk in 680000 people exposed to computed tomography scans in childhood or adolescence: data linkage study of 11 million Australians," BMJ, vol. 346, pp. 1-18, 2013.

[13] C. Cipriano, A. M. Griffin, P. C. Ferguson, and J. S. Wunder, "Developing an evidence-based followup schedule for bone sarcomas based on local recurrence and metastatic progression," Clinical Orthopaedics and Related Research, vol. 475, no. 3, pp. 830-838, 2017.

[14] P. G. Casali, S. Bielack, N. Abecassis et al., "Bone sarcomas: ESMO-PaedCan-EURACAN Clinical Practice Guidelines for diagnosis, treatment and follow-up," Annals of Oncology: Official Journal of the European Society for Medical Oncology, vol. 29, no. Suppl 4, pp. iv79-95, 2018.

[15] A. Puri, P. Ranganathan, A. Gulia, S. Crasto, R. Hawaldar, and R. A. Badwe, "Does a less intensive surveillance protocol affect the survival of patients after treatment of a sarcoma of the limb?" The Bone \& Joint Journal, vol. 100-B, no. 2, pp. 262-268, 2018.

[16] NICE guidance, "Improving outcomes for people with sarcoma,” 2006, https://www.nice.org.uk/guidance/csg9/ evidence/full-guideline-pdf-2188960813.

[17] P. Cool, R. Grimer, and R. Rees, "Surveillance in patients with sarcoma of the extremities," European Journal of Surgical Oncology (EJSO), vol. 31, no. 9, pp. 1020-1024, 2005.

[18] F. Chen, R. Miyahara, T. Bando et al., "Prognostic factors of pulmonary metastasectomy for osteosarcomas of the extremities," European Journal of Cardio-Thoracic Surgery, vol. 34, no. 6, pp. 1235-1239, 2008.

[19] Y. Yamamoto, R. Kanzaki, T. Kanou et al., "Long-term outcomes and prognostic factors of pulmonary metastasectomy for osteosarcoma and soft tissue sarcoma," International Journal of Clinical Oncology, vol. 24, no. 7, pp. 863-870, 2019.

[20] C. E. García Franco, W. Torre, A. Tamura et al., "Long-term results after resection for bone sarcoma pulmonary metastases," European Journal of Cardio-Thoracic Surgery, vol. 37, no. 5, pp. 1205-1208, 2010.

[21] P. Cool and G. Cribb, "The impact and efficacy of surveillance in patients with sarcoma of the extremities," Bone \& Joint Research, vol. 6, no. 4, pp. 224-230, 2017.

[22] W. Kim, I. Han, J. S. Lee, H. S. Cho, J. W. Park, and H.-S. Kim, "Postmetastasis survival in high-grade extremity osteosarcoma: a retrospective analysis of prognostic factors in 126 patients," Journal of Surgical Oncology, vol. 117, no. 6, pp. 1223-1231, 2018.

[23] C. Gerrand, N. Athanasou, B. Brennan et al., "UK guidelines for the management of bone sarcomas," Clinical Sarcoma Research, vol. 6, no. 1, 2016.

[24] N. M. Marina, L. Packard, Q. Liu et al., "Longitudinal followup of adult survivors of Ewing sarcoma: a report from the childhood cancer survivor study," Cancer, vol. 123, pp. 2551-2560, 2017.

[25] V. M. van Praag, A. J. Rueten-Budde, L. M. Jeys et al., "A prediction model for treatment decisions in high-grade extremity soft-tissue sarcomas: personalised sarcoma care (PERSARC)," European Journal of Cancer, vol. 83, pp. 313323, 2017. 